\section{Triaging deep vein thrombosis using ultrasonography after lower-extremity orthopedic surgery: analysis of a single-center experience}

\author{
Hee Joong Lim', Ji Young Jeon', Yu Mi Jeong', Beom Gu Lee², Jae-Ang Sim², \\ Sheen-Woo Lee ${ }^{1,3}$
}

Departments of ${ }^{1}$ Radiology and ${ }^{2}$ Orthopedic Surgery, Gachon University Medical Center, Gachon University College of Medicine, Incheon; ${ }^{3}$ Department of Radiology, Eunpyeong St. Mary's Hospital, College of Medicine, The Catholic University of Korea, Seoul, Korea

Purpose: This study aimed to stratify risk factors and vein levels for postoperative deep vein thrombosis (DVT) after lower-extremity orthopedic surgery.

Methods: Ninety-nine patients who underwent Doppler ultrasonography after lower-extremity orthopedic surgery were enrolled. Medical records were reviewed for anesthesia duration, type of surgery, body weight, height, and cardiovascular risk factors (including history of smoking, diabetes mellitus or hypertension, blood pressure, and total cholesterol and high-density lipoprotein [HDL] cholesterol levels), and the DVT treatment. Ultrasound diagnosis of DVT was made according to a routine protocol. The relationships between selected factors and the presence of DVT were assessed using univariate and multivariate regression analyses.

Results: Thirty-three (33\%) patients were found to have calf DVT. The mean age, weight, and height of the non-DVT and postoperative DVT patients were 55.1 years versus 65.4 years, 70.5 $\mathrm{kg}$ versus $61.2 \mathrm{~kg}$, and $163.3 \mathrm{~cm}$ versus $157.0 \mathrm{~cm}$, respectively. Total cholesterol/HDL levels in the non-DVT and DVT patients were 70.6/20.7 mg/dL and $90.8 / 26.0 \mathrm{mg} / \mathrm{dL}$, retrospectively. Systolic and diastolic blood pressure in the non-DVT and DVT patients were 133.6/80.2 mm Hg and $132.2 / 78.1 \mathrm{mmHg}$, respectively. The mean duration of anesthesia was 173.9 versus 199.9 minutes, and the operative time was 136.4 minutes versus 161.0 minutes. Older age $(P=0.005)$ and lower body weight ( $\mathrm{P}=0.002$ ) were significantly associated with postoperative DVT. No other significant between-group differences were found $(P>0.05)$. The patients with ultrasoundidentified DVT received antithrombotic treatment. None of them had distant thromboembolism. Conclusion: After lower-extremity orthopedic surgery, the calf veins in elderly patients with low body weight are susceptible to thrombosis; they would most likely benefit from postoperative ultrasonography.

Keywords: Deep vein thrombosis; Ultrasound; Postoperative; Risk factor; Orthopedics

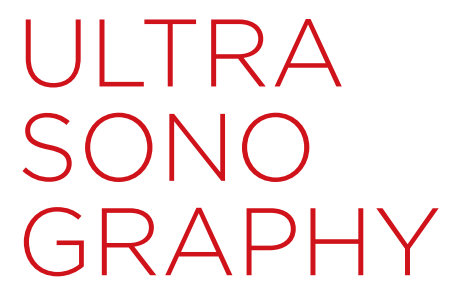

ORIGINAL ARTICLE

https://doi.org/10.14366/usg.20171 pISSN: 2288-5919 - elSSN: 2288-5943 Ultrasonography 2021;40:442-448

Received: October 29, 2020

Revised: December 27, 2020

Accepted: January 12, 2021

Correspondence to:

Sheen-Woo Lee, MD, Department of Radiology, Eunpyeong St. Mary's Hospital, College of Medicine, The Catholic University of Korea, 1021 Tongil-ro, Eunpyeong-gu, Seoul 03312, Korea

Tel. +82-2-2030-3016

Fax. +82-2-2030-2965

E-mail: leesw1@gmail.com

This is an Open Access article distributed under the terms of the Creative Commons Attribution NonCommercial License (http://creativecommons.org/ licenses/by-nc/4.0/) which permits unrestricted noncommercial use, distribution, and reproduction in any medium, provided the original work is properly cited.

Copyright (C) 2021 Korean Society of Ultrasound in Medicine (KSUM)

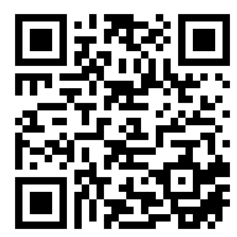

How to cite this article:

Lim HJ, Jeon JY, Jeong YM, Lee BG, Sim JA, Lee SW. Triaging deep vein thrombosis using ultrasonography after lower-extremity orthopedic surgery: analysis of a singlecenter experience. Ultrasonography. 2021 Jul:40(3):442-448. 


\section{Introduction}

Deep vein thrombosis (DVT) occurs when a thrombus forms in one or more deep veins. It is a common clinical problem and usually affects the lower limbs; moreover, orthopedic surgery is a known risk factor for DVT [1]. DVT can lead to pulmonary thromboembolism or post-thrombotic syndrome, which can result in death. Therefore, orthopedic surgeons usually plan to identify DVT in the lower extremities. Although it is well known that ultrasonography is an excellent modality for postoperative evaluations of orthopedic patients, and venous Doppler ultrasonography is the standard imaging modality for patients with suspected DVT, the procedure can be limited due to issues with the acoustic window and patient discomfort after surgery $[2,3]$. Moreover, computed tomography angiography (CTA) of the femoral vein is an alternative imaging modality, but involves potentially harmful radiation exposure, the administration of iodinated contrast agents, and a higher degree of invasiveness than ultrasonography. Therefore, it would be helpful to prioritize patients with significant risk factors who would truly benefit from postoperative ultrasonography.

The presence of DVT is associated with multiple factors. A previous study suggested that prolonged surgery ( $\geq 2$ hours) and a higher body mass index were significantly associated with the development of DVT in patients undergoing major orthopedic surgery [4]. Another study reported that major orthopedic surgery was associated with a greater risk for DVT [5]. However, contradictory studies have also reported that cardiovascular disease (CVD) risk factors, such as age, smoking, adiposity, cholesterol and triglyceride levels, and diabetes mellitus (DM), were associated or not associated with venous thromboembolism $[6,7]$. The risk factors associated with lowerlimb surgery, especially in elderly patients undergoing total knee joint replacement, and controversies regarding the prevalence of DVT, site, and host-related factors, have led to uncertainty regarding which patients require a more rigorous work-up for venous thromboembolism.

Accordingly, the objectives of the present study were to verify which patients need post-orthopedic surgery examinations for DVT by identifying the factors associated with post-orthopedic surgery DVT at our hospital and to determine whether any specific level of vessel is more vulnerable to DVT after lower-extremity surgery in order to help triage patient management in the long run.

\section{Materials and Methods}

Design, Sample Population, and Inclusion/Exclusion Criteria This retrospective cohort study used data extracted from medical records and case information. The data remained anonymized, and no patients were directly involved in setting the research questions or the outcome measures, nor were they involved in the recruitment or implementation of the study. The requirement for informed consent was waived due to the retrospectively used medical records. It was approved by the Institutional Review Board (GDIRB 2020-330) and was conducted at Gachon University Gil Hospital (Incheon, Korea) between January 2018 and December 2019. A total of 104 potentially eligible patients, who were referred for an ultrasound work-up for suspected DVT after orthopedic surgery, and for whom the orthopedic surgeon requested ultrasonography and not computed tomography (CT) for postoperative DVT evaluation during the study period, were included. Individuals with confirmed DVT before surgery and those who underwent surgery of the upper extremities were excluded. As such, 99 patients were ultimately included in the present study.

\section{DVT Diagnosis}

All DVT examinations were performed using a linear phased-array transducer (EPIQ 7G, Philips Healthcare, Best, Netherlands) by one of eight radiology residents under the supervision of one of four musculoskeletal radiologists. The examination followed a set protocol irrespective of the examiner's expertise, using the twodimensional compression technique in the supine or prone position throughout the leg, tracing the deep veins from the inguinal ligament to the femoropopliteal and tibioperoneal trunk on the transverse plane. A 3- or 5-MHz probe was applied, depending on the patient's anatomy and the adequate depth of penetration for an optimal examination of the deep veins. Color Doppler imaging was used as a road map and only if the calf veins were inadequately visualized on real-time imaging alone. We diagnosed DVT with comprehensive duplex ultrasound combined with Doppler rather than a limited or complete compression-only examination. The mean scanning time was 10 minutes per patient. The diagnostic criteria for DVT were incompressibility of the vein with probe pressure in the affected part, and an absence of flow signal in the venous thrombosed segments (Fig. 1). This technique is routinely performed at many hospitals and can be carried out using standard ultrasound equipment and personnel. The patients were divided into a DVT group ( $n=33)$ and a non-DVT group $(n=66)$.

\section{Data Collection and Statistical Analysis}

Data were collected on age, sex, height, body weight, duration of anesthesia, type of orthopedic surgery, and CVD risk factors. We enumerated the risk factors of CVD according to the Framingham risk score, which is a 10-year CVD risk score model that reliably predicts CVD events and mortality in the next 10 years in subjects with baseline examinations free of CVD. The predictors used in 
the risk model included age, sex, total cholesterol, high-density lipoprotein (HDL) cholesterol, systolic blood pressure (BP), treatment status of hypertension or diabetes, and smoking status. All data were collected from patients' medical records and laboratory investigations. Statistical analysis of the data was done using the chi-square test, independent-sample $t$ test, and logistic regression analysis in SPSS version 22.0 (IBM Corp., Armonk, NY, USA) for Windows (Microsoft Corporation, Redmond, WA, USA). Differences with a P-value $<0.05$ were considered to be statistically significant.

\section{Results}

\section{Patient Information}

The demographic characteristics of all individuals enrolled in the present study, including age, sex, height, body weight, duration of anesthesia, and operative time, are summarized in Table 1. The types of orthopedic surgery performed included total knee replacement arthroplasty $(n=40)$, ligament reconstruction $(n=14)$, meniscectomy $(n=17)$, open reduction and internal fixation $(n=17)$, incision and drainage $(n=8)$, diagnostic arthroscopy $(n=1)$, and others including tendon repair $(n=2)$. Medical information regarding known CVD risk is presented in Table 2. Among 99 post-orthopedic subjects, 33 had DVT (seven with a history of surgery); the thrombosis was at the popliteal vein in only one patient, while it was below the popliteal vein in the other 32 patients. The mean age, weight, and height of the non-DVT and postoperative DVT patients was $55.1 \pm 17.9$ years and $65.4 \pm 12.6$ years, $70.5 \pm 15.6 \mathrm{~kg}$ and $61.2 \pm 8.1 \mathrm{~kg}, 163.3 \pm 11.7 \mathrm{~cm}$ and $157.0 \pm 7.2 \mathrm{~cm}$, and $26.2 \mathrm{~kg} / \mathrm{m}^{2}$ and $24.8 \mathrm{~kg} / \mathrm{m}^{2}$, respectively. Total

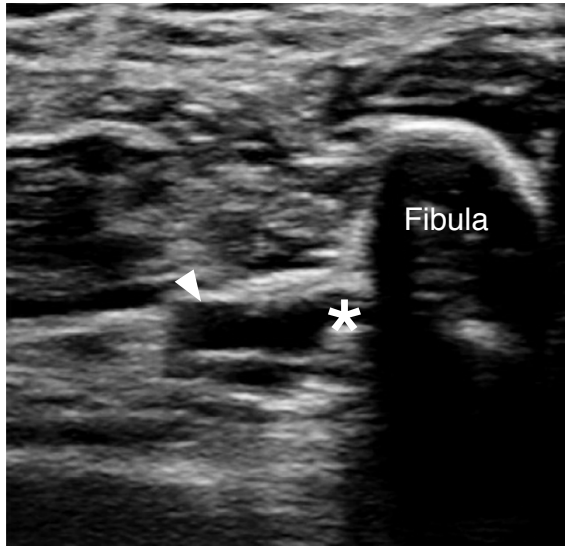

A

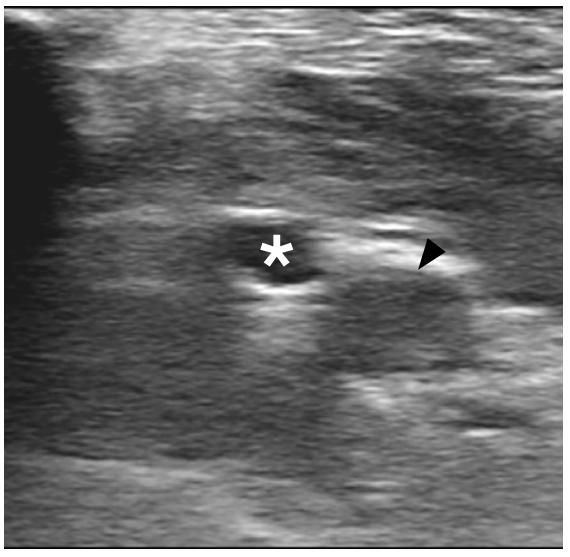

D

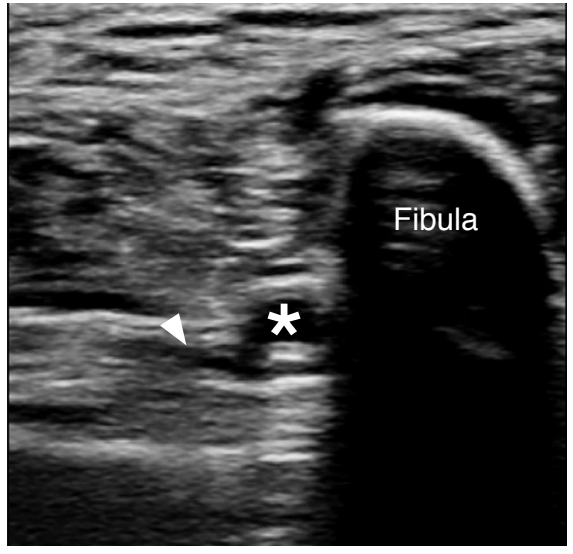

B

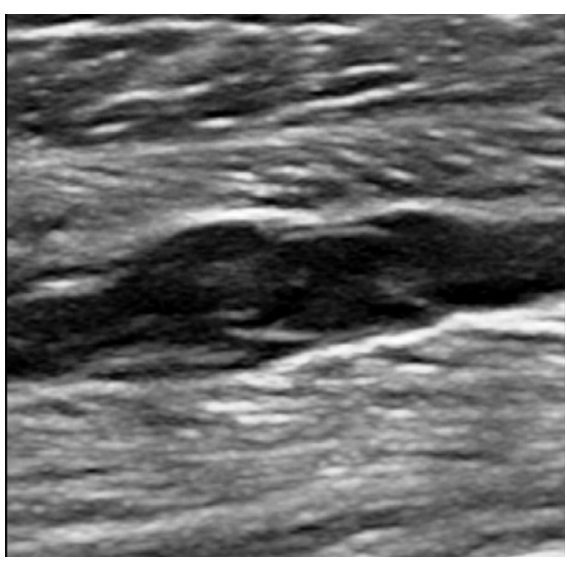

E

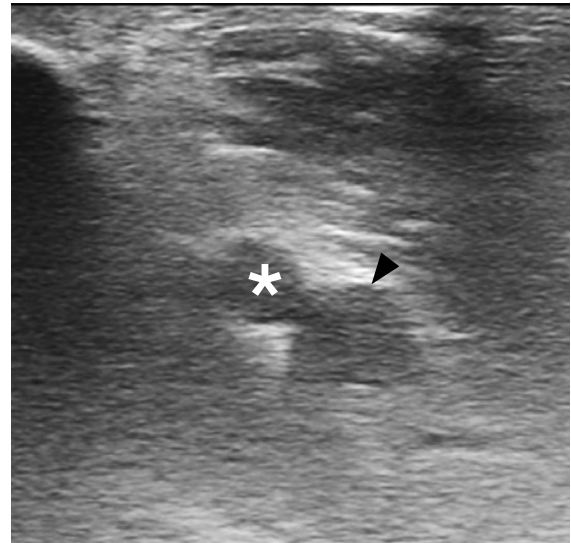

C

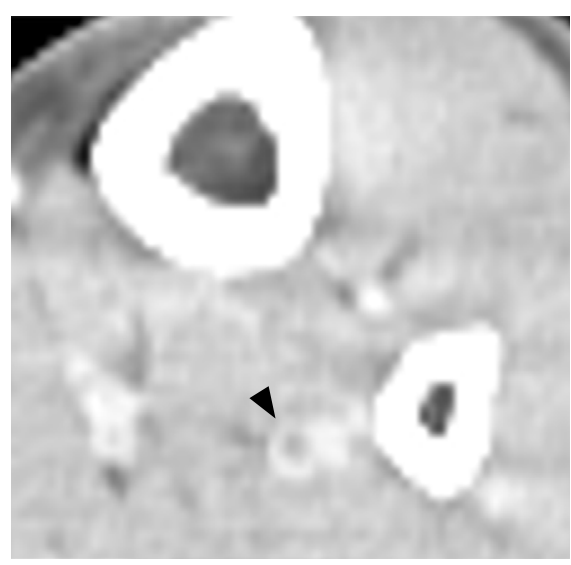

$\mathrm{F}$

Fig. 1. Normal findings versus a thrombus in the peroneal veins.

$A, B$. Side-by side axial gray-scale ultrasonography of the calf without (A) and with (B) the manual compression maneuver shows anechoic peroneal veins (arrowhead) adjacent to the peroneal artery (asterisk) and fibula. After compression, the peroneal veins are compressed (arrowhead), indicating the absence of a thrombus. C-E. Side-by-side non-compressed and compressed gray-scale axial (C, D), and long-axis (E) images visualize echogenic material filling the peroneal veins (arrowhead). Asterisk indicates peroneal artery. E, F. After compression, the veins do not collapse. Femoral computed tomography angiography of the same patient (F) shows the absence of contrast within the venous lumen (arrowhead), indicating the presence of a thrombus. 
Table 1. Demographic information from 99 patients who underwent Doppler ultrasonography after lower-extremity orthopedic surgery

\begin{tabular}{lccc}
\hline \multicolumn{1}{c}{ Parameter } & DVT $(\mathrm{n}=33)$ & Non-DVT $(\mathrm{n}=66)$ & P value \\
\hline Age (year) & $65.4 \pm 12.6$ & $55.1 \pm 17.9$ & 0.005 \\
Sex & & & 0.243 \\
$\quad$ Male & $5(13.9)$ & $31(86.1)$ & \\
$\quad$ Female & $28(44.4)$ & $35(55.6)$ & \\
Height (cm) & $157.0 \pm 7.2$ & $163.3 \pm 11.7$ & 0.050 \\
Body weight (kg) & $61.2 \pm 8.1$ & $70.5 \pm 15.6$ & 0.002 \\
Anesthesia (min) & $173.2 \pm 75.3$ & $158.9 \pm 60.9$ & 0.236 \\
Operation (min) & $135.5 \pm 72.1$ & $122.7 \pm 56.1$ & 0.258
\end{tabular}

*Values are presented as mean \pm SD or number (\%).

DVT, deep vein thrombosis; SD, standard deviation.

cholesterol, HDL, and systolic and diastolic BP were $70.6 \pm 87.6 \mathrm{mg} /$ $\mathrm{dL}$ and $90.8 \pm 93.1 \mathrm{mg} / \mathrm{dL}, 20.7 \pm 25.9 \mathrm{mg} / \mathrm{dL}$ and $26.0 \pm 26.8 \mathrm{mg} / \mathrm{dL}$, and $133.6 / 80.2 \pm 15.7 / 9.9 \mathrm{mmHg}$, and $132.2 / 78.1 \pm 13.9 / 8.6 \mathrm{~mm}$ $\mathrm{Hg}$, respectively. The mean duration of anesthesia was $158.9 \pm 60.9$ minutes and $173.2 \pm 75.3$ minutes, and the operative time was $122.7 \pm 56.1$ minutes and $135.5 \pm 72.1$ minutes, respectively. All patients with DVT received intermittent pneumatic compression and were initially managed with apixaban or rivaroxaban. The patients were discharged with short-term follow-up at the outpatient clinic.

\section{Univariate and Multivariate Analyses of Risk Factors}

Univariate logistic regression analysis indicated that two variables were significantly associated with the development of DVT: older age $(P<0.05)$ and lower body weight $(P<0.05)$. In contrast, total cholesterol, HDL, systolic and diastolic BP, mean duration of anesthesia, and operative time were not significantly correlated with postoperative DVT, although there was a rising trend in the incidence of DVT with increasing values in each category. Multivariate logistic regression also revealed the same result with regard to age and body weight $(P<0.05)$. The risk of DVT associated with surgery increased with age and decreased with body weight (odds ratio [OR], 3.3 if $>60$ years; $95 \%$ confidence interval $[\mathrm{Cl}], 1.3$ to $8.5 ; \mathrm{OR}, 3.1$ if $<60 \mathrm{~kg} ; 95 \% \mathrm{Cl}, 1.3$ to $7.3 ; \mathrm{P}<0.05)$.

\section{Discussion}

Orthopedic surgery is a well-known risk factor for DVT. The release of thromboplastin from dissected soft tissue and reamed bone, as well as venous stasis during surgery and postoperative immobility, contribute to high rates of DVT [8]. The frequency of all major lower-limb orthopedic surgical procedures has increased annually, similar to what has been reported in previous studies [5]. This study
Table 2. Medical information obtained for known cardiovascular risk factors

\begin{tabular}{lccc}
\multicolumn{1}{c}{ Parameter } & DVT $(\mathrm{n}=33)$ & Non-DVT $(\mathrm{n}=66)$ & P value \\
\hline Total cholesterol & $90.8 \pm 93.1$ & $70.6 \pm 87.6$ & 0.517 \\
$(\mathrm{mg} / \mathrm{dL})$ & $26.0 \pm 26.8$ & $20.8 \pm 25.9$ & 0.611 \\
$\mathrm{HDL}(\mathrm{mg} / \mathrm{dL})$ & $132.2 \pm 13.9$ & $133.6 \pm 15.7$ & 0.710 \\
Systolic BP $(\mathrm{mmHg})$ & $\mathbf{1 3}$ & $80.2 \pm 9.9$ & 0.548 \\
Diastolic BP $(\mathrm{mmHg})$ & $78.1 \pm 8.6$ & $28(68.3)$ & 0.631 \\
Hypertension & $13(31.7)$ & $10(83.3)$ & 0.188 \\
Smoking & $2(16.7)$ & $13(68.4)$ & 0.702 \\
\hline Diabetes mellitus & $6(31.6)$ & &
\end{tabular}

*Values are presented as mean \pm SD or number (\%).

DVT, deep vein thrombosis; HDL, high-density lipoprotein; BP, blood pressure.

investigated the incidence and causes of postoperative DVT, which has multifactorial risk factors. We found that approximately a third of postoperative patients had DVT distal to the knee, and DVT was significantly associated with older age and lower body weight.

Ultrasonography plays a role as an important imaging modality in the accurate diagnosis of postoperative DVT. Most of all, this imaging modality is not affected by metal artifacts, which is especially relevant after musculoskeletal surgery. The susceptibility of magnetic resonance imaging to artifacts, as well as the presence of streak artifacts in $\mathrm{CT}$, can reduce the diagnostic performance of these modalities for DVT. Second, the flexibility of ultrasound examinations in a variety of locations and patients positions is a strength in the postoperative setting. Ultrasonography at various angles may also help overcome the poor sonic window. Third, ultrasonography is a fast, reliable, and cost-effective technique compared to other modalities. Moreover, it can be used to evaluate postoperative pathologic conditions, such as abscesses, muscles or tendon tears, or the extent of hemarthrosis [2].

A previous report showed that knee surgery had a higher incidence of DVT at the localized popliteal location [9]. Unlike the previous study, most DVT sites in our cases were distal to the popliteal vein, including the tibial, peroneal, soleus, (Fig. 2) and gastrocnemius veins; only one DVT was found at the level of the popliteal vein, and no patients had distant thromboembolism. Calf vein thromboses have been reported to be closely related with lack of limb movement. More specifically, the soleal intramuscular veins depend on muscle contraction for venous perfusion, and their valve system does not function sufficiently in immobile patients, so that these veins are the leading points of DVT in the leg. Therefore, postoperative patients, especially after lower-limb surgery, are susceptible to venous stasis due to lack of motion. Our data showed 


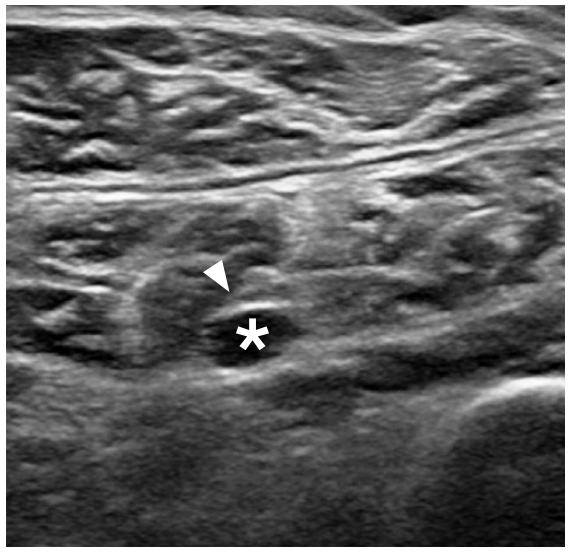

A

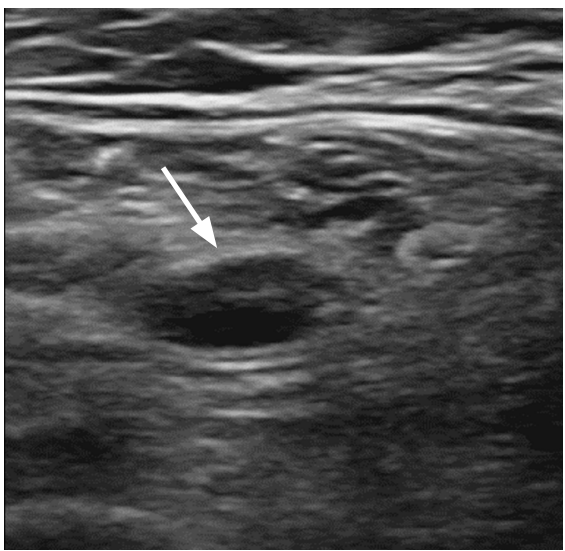

D

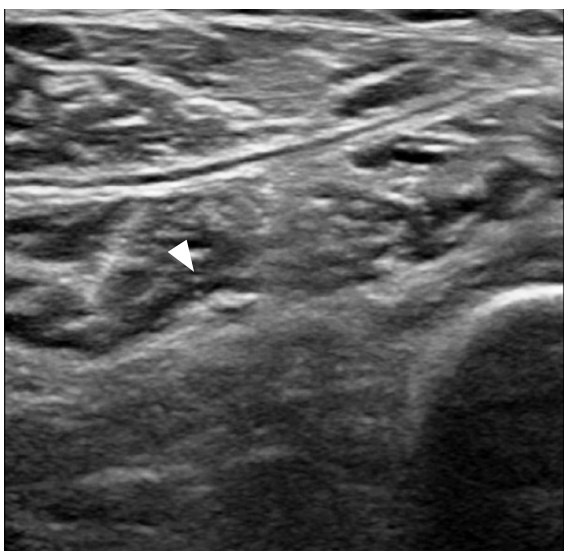

B

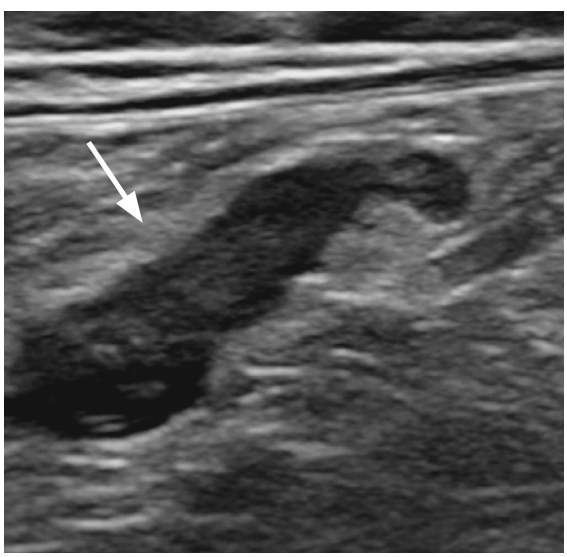

$\mathrm{E}$

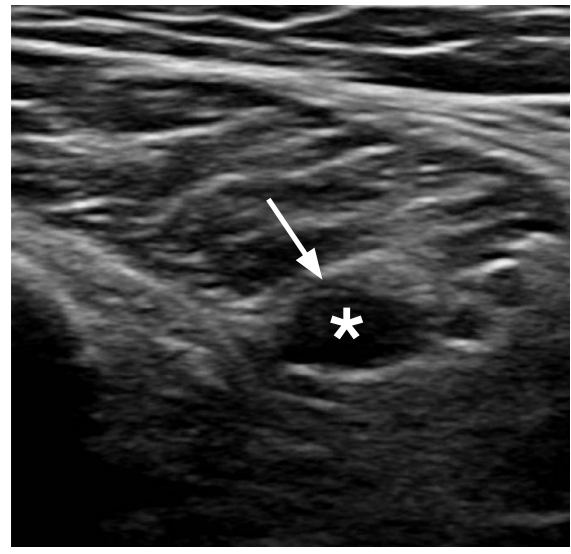

C

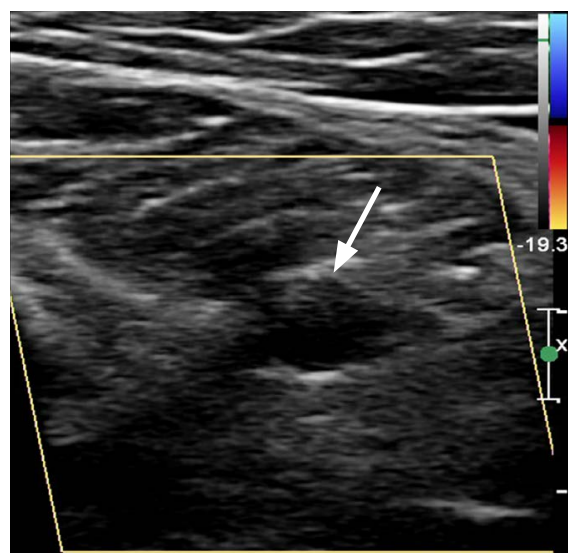

$\mathrm{F}$

Fig. 2. Normal findings versus acute deep vein thrombosis in the soleal vein.

A, B. Non-compressed (A) and compressed (B) gray-scale axial gray-scale ultrasonography shows an anechoic soleal vein (asterisk), which is collapsed on compression (arrowhead), indicating no evidence of deep vein thrombosis. C-F. Non-compressed (C) and compressed axial and long-axis gray-scale (D,E) and color Doppler (F) images show the typical ultrasonographic findings of soleal intramuscular thrombus (arrows).

the typical location of DVTs in this context. In contrast, iliac or femoral vein lesions are mainly caused by iliac vein compression, or catheter-related injury, and are symptomatic.

The major threat of a soleal thrombus is that it can propagate silently to the thigh, and form a freely floating distant thromboembolism to the lung. Therefore, it is important to remain vigilant for calf lesions, especially in patients with a known risk for DVT [10-12].

The risk factors known to be related with DVT were analyzed in our study, and interestingly, instead of the usual suspects (e.g., prolonged surgery time or cardiovascular risk factors), we found that lower body weight was associated with a higher incidence of postoperative DVT. In one study, the relative muscle volume of the vastus lateralis at the level of the thigh was negatively associated with the prevalence of symptomatic and radiologically detected DVT [13]. Lower body weight can be accompanied by loss of muscle volume, which is known as sarcopenia. Sarcopenia is defined as the loss of skeletal muscle mass and strength with aging, and is a recognized component of geriatric syndrome [14]. It has been associated with a multitude of poor outcomes including low survival rate, high infection rate, prolonged hospital stay, treatment toxicity, and physical disability [15]. Although obesity is a known risk factor for DVT, it is uncertain whether only a higher body weight is an independent risk factor for DVT, or whether the effect is secondary to DM and dyslipidemia [16]. We suggest future research on sarcopenia, especially in the leg, which may be related to the occurrence of DVT.

In addition, low muscle mass has also negative correlations with an increased prevalence of coronary arterial disorder(s) and metabolic syndrome [17]. Future studies investigating the associations of DVT with the Framingham risk score and leg muscle mass are warranted.

The management of below-knee DVT remains inconsistent 
because current guidelines are based on weak evidence. The American College of Chest Physicians recommends anticoagulation if symptoms are severe; otherwise, it is recommended to perform surveillance ultrasonography 2 weeks after surgery to monitor for clot propagation, at which point anticoagulation should be initiated if there is any evidence of proximal extension [18]. Patient symptoms and a D-dimer test in the early postoperative period, however, may lead to clinical confusion and mask the thrombosis. Therefore, it is the responsibility of radiologists to clearly report sonographic findings in a way that most appropriately facilitates patient management, and recommend an alternative study such as CTA in cases of severe postoperative changes in overlying soft tissue shadowing the deep venous structure. When postoperative ultrasonography for DVT showed the presence of a thrombosis, the clinicians at our hospital treated the respective patients with antithrombotic agents, even for isolated calf vein lesions, closely monitored them at the outpatient clinic, and followed up with ultrasonography. Most DVT sites in our patients were distal to the popliteal vein, with multiple sites, including the tibial vein in five patients, the peroneal vein in 13 patients, the soleal vein in 28 patients, and the gastrocnemius veins in 25 patients. None of them had adverse events related with anticoagulation therapy or distant thromboembolism. All showed resolution of the thrombosis on follow-up. We believe that early sonographic recognition and treatment may have prevented thrombotic extension or recurrence, although this possibility needs future study.

Several limitations of this study should be acknowledged. First, data analysis was performed retrospectively at a single tertiary referral center, and some asymptomatic patients may have missed the postoperative evaluation. Second, DVT ultrasonography is considered to be an operator-dependent imaging procedure. The variable expertise of the operators may have affected the reported incidence of DVT. To decrease inter-operator variability, the study was performed using a standardized protocol, and the expert musculoskeletal radiologists confirmed the examinations. Additionally, with more expert examinations, the incidence of distal DVT might even increase, since it is more difficult to diagnose a thrombosis in a calf vein than at the femoropopliteal level in postoperative patients [19]. Third, DVT prophylaxis regimens and prevention programs may have affected the outcomes of our study.

In conclusion, isolated distal DVTs were found to be common in patients after lower-limb surgery. We showed that advanced age and lower body weight were associated with a higher incidence of postoperative DVT. The typical location was below the popliteal vein, which may be related to venous stasis. Therefore, physicians should carefully examine the calf level, especially in elderly and fragile patients. In the aging society, where more and more elderly patients with DVT risk factors undergo major surgery, we hope that the typical ultrasonography findings shown in our study will help radiologists screen at-risk patients.

ORCID: Hee Joong Lim: https://orcid.org/0000-0002-9931-6472; Ji Young Jeon: https://orcid.org/0000-0002-9864-4765; Yu Mi Jeong: https://orcid.org/0000-00023880-0156; Beom Gu Lee: https://orcid.org/0000-0002-7293-1379; Jae-Ang Sim: https://orcid.org/0000-0002-2055-6458; Sheen-Woo Lee: https://orcid.org/00000002-7861-7153

\section{Author Contributions}

Conceptualization: Lee BG. Data acquisition: Lim HJ, Lee SW, Jeong YM, Sim JA. Data analysis or interpretation: Lim HJ, Lee SW, Jeon JY. Drafting of the manuscript: Lim HJ, Lee SW. Critical revision of the manuscript: Jeon JY, Jeong YM, Lee BG, Sim JA. Approval of the final version of the manuscript: all authors.

\section{Conflict of Interest}

No potential conflict of interest relevant to this article was reported.

\section{References}

1. Monreal M, Ruiz J, Olazabal A, Arias A, Roca J. Deep venous thrombosis and the risk of pulmonary embolism: a systematic study. Chest 1992;102:677-681.

2. Chun KA, Cho KH. Postoperative ultrasonography of the musculoskeletal system. Ultrasonography 2015;34:195-205.

3. Gobl R, Virga S, Rackerseder J, Frisch B, Navab N, Hennersperger C. Acoustic window planning for ultrasound acquisition. Int I Comput Assist Radiol Surg 2017;12:993-1001.

4. Bagaria V, Modi N, Panghate A, Vaidya S. Incidence and risk factors for development of venous thromboembolism in Indian patients undergoing major orthopaedic surgery: results of a prospective study. Postgrad Med J 2006;82:136-139.

5. Lee SY, Ro du H, Chung CY, Lee KM, Kwon SS, Sung KH, et al. Incidence of deep vein thrombosis after major lower limb orthopedic surgery: analysis of a nationwide claim registry. Yonsei Med J 2015;56:139-145.

6. Holst $A G$, Jensen $G$, Prescott E. Risk factors for venous thromboembolism: results from the Copenhagen City Heart Study. Circulation 2010;121:1896-1903.

7. Mahmoodi BK, Cushman M, Anne Naess I, Allison MA, Bos WJ, Braekkan SK, et al. Association of traditional cardiovascular risk factors with venous thromboembolism: an individual participant data meta-analysis of prospective studies. Circulation 2017;135:716.

8. Warwick D. Prevention of venous thromboembolism in total knee and hip replacement. Circulation 2012;125:2151-2155.

9. Davidson HC, Mazzu D, Gage BF, Jeffrey RB. Screening for deep 
venous thrombosis in asymptomatic postoperative orthopedic patients using color Doppler sonography: analysis of prevalence and risk factors. AJR Am J Roentgenol 1996;166:659-662.

10. Ro A, Kageyama N. Clinical significance of the soleal vein and related drainage veins, in calf vein thrombosis in autopsy cases with massive pulmonary thromboembolism. Ann Vasc Dis 2016;9:15-21.

11. Ro A, Kageyama N, Mukai T. Pathophysiology of venous thromboembolism with respect to the anatomical features of the deep veins of lower limbs: a review. Ann Vasc Dis 2017;10:99-106.

12. Utter GH, Dhillon TS, Salcedo ES, Shouldice DJ, Reynolds CL, Humphries MD, et al. Therapeutic anticoagulation for isolated calf deep vein thrombosis. JAMA Surg 2016;151:e161770.

13. Shin JM, Hong SJ, Choi KH, Shin SI, Lee DK, Lee SS, et al. Low relative muscle volume: correlation with prevalence of venous thromboembolism following total knee arthroplasty. PLoS One 2019;14:e0210800.

14. Cruz-Jentoft AJ, Baeyens JP, Bauer JM, Boirie Y, Cederholm T, Landi F, et al. Sarcopenia: European consensus on definition and diagnosis: report of the European Working Group on Sarcopenia in Older People. Age Ageing 2010;39:412-423.
15. Taguchi S, Akamatsu N, Nakagawa T, Gonoi W, Kanatani A, Miyazaki $\mathrm{H}$, et al. Sarcopenia evaluated using the skeletal muscle index is a significant prognostic factor for metastatic urothelial carcinoma. Clin Genitourin Cancer 2016;14:237-243.

16. Darvall KA, Sam RC, Silverman SH, Bradbury AW, Adam DJ. Obesity and thrombosis. Eur J Vasc Endovasc Surg 2007;33:223-233.

17. Ko BJ, Chang Y, Jung HS, Yun KE, Kim CW, Park HS, et al. Relationship between low relative muscle mass and coronary artery calcification in healthy adults. Arterioscler Thromb Vasc Biol 2016;36:1016-1021.

18. Kearon C, AkI EA, Comerota AJ, Prandoni P, Bounameaux H, Goldhaber SZ, et al. Antithrombotic therapy for VTE disease: antithrombotic therapy and prevention of thrombosis, 9th ed: American College of Chest Physicians evidence-based clinical practice guidelines. Chest 2012;141:e4195-e496S.

19. Atri M, Herba MJ, Reinhold C, Leclerc J, Ye S, Illescas FF, et al. Accuracy of sonography in the evaluation of calf deep vein thrombosis in both postoperative surveillance and symptomatic patients. AJR Am J Roentgenol 1996;166:1361-1367. 